\title{
Finite Element Analysis Of thin Quartz Wafer Stress Distribution In The
}

\section{Radial Force}

\author{
Wenjie Tian ${ }^{1, a,{ }^{*}}$, Qinjiang Zhao ${ }^{2, b}$, Sanming Feng ${ }^{1, c}$, Bo Ma ${ }^{1, d}$ \\ ${ }^{1}$ Key Laboratory for Sensor, Beijing Information Science \& Technology University, \\ Beijing ,100101,China ; \\ 2 Department of Physical Science and Technology, Kunming University, \\ Kunming , 650214, China \\ a twjkm2008@163.com, bzqj00213@163.com , c996613627@qq.com , d18903431824@163.com \\ ${ }^{*}$ corresponding author
}

Key words: radial force; thin circular quartz crystal; stress distribution; finite element analysis Abstract. Extending the stress distribution result in semi-infinite anisotropic media slice with centralized force to thin circular quartz crystal resonator, the relationship of frequency relative variation and adding force azimuth and adding force strength of the resonator with radial centralized force was obtained, and the result of comparing with the relative frequency variation of the resonator with electrodes on its geometric center was also gained. At the same time, using the finite element method analyzed the stress variation in the different positions on the thin circular quartz crystal slice with radial force, and deposited electrode in the correspond position to form resonator, it's force-sensitive character in different positions was tested. The result shows, the theoretical calculations and analysis was consistent with the experimental result.

\section{Introduction}

Quartz crystal resonator has been widely used in the field of the frequency selection and homeostasis control due to its high quality factor. At the same time the properties (such as temperature characteristics, force sensing properties) related with quartz-cut have also been a great concern and have made a lot of theoretical and experimental studies ${ }^{[1-7]}$ in these areas. A function about the amount of frequency change of quartz wafer, afterburner azimuth and rotation angle-cut (Y-cut family) in thickness shear vibration has been got $^{[8]}$. And under certain conditions, the approximate solution of quartz anisotropic wafer's stress distribution has been obtained too ${ }^{[9]}$.

When the thin wafer-shaped quartz crystal resonator that has a certain cut type is used as the force sensor for sensing system, resonator will be combined with the certain conversion structures. The measured is converted to power through this conversion structure. And it is applied to the resonator in diameter direction to make the internal stress of resonator crystal change. Finally, the resonant frequency will change. Using this relationship, we developed a series of quartz detection and control systems, such as force, pressure, posture, acceleration, quality and other sensing system. So to analyze the stress variation and change inside the crystal on the thin circular quartz crystal resonator with radial force has far-reaching theoretical significance and practical value for further 
improving the resonance theory and extending quartz crystal resonator applications.

\section{The Stress Distribution Of A Thin Disk-shaped Crystal}

To the thin circular quartz crystal, the results of semi-infinite anisotropic medium's internal stress distribution can been applied to this structure when the concentrated force acts on the flakes by using the edge structure and cyclical characteristic. When the radial force $\boldsymbol{F}$ is applied to a thin disk-shaped quartz crystal, the relationship between the crystal internal stress $\sigma_{1}, \sigma_{2}, \sigma_{r}, \sigma_{\theta}$ and coordinate can been described by the mechanical model of Fig.1.

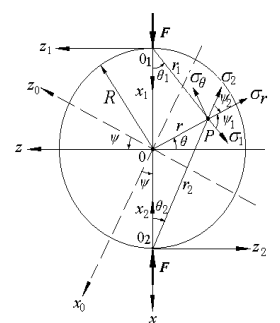

Fig.1 the stress model of quartz crystal flakes under the radial force

In Fig.1, the thickness of a circular wafer is much thinner than the diameter, so the wafer can be viewed as a two-dimensional plane and it is parallel to the drawing. Force coordinates $x o z$ is within the wafer plane; $o x$ axis and the radial force $\boldsymbol{F}$ suffered on the wafer are collinear; oy axis along the wafer thickness direction is perpendicular to the paper. Its direction is upward and positive. $x_{0} \mathrm{O} z_{0}$ is crystal coordinates; For AT-cut circular quartz wafer, $o x_{0}$ axis is the electrical axis of the crystal, $\boldsymbol{R}$ is the radius of the crystal wafer, $\psi$ is the adding force azimuth (i.e., the angle between the direction of adding force and $\mathrm{o} x_{0}$ axis). $\mathrm{o}_{1}, \mathrm{o}_{2}$ are the force acting points of radial force $\boldsymbol{F}$. In these two positions, $x_{1} \mathrm{O}_{1} z_{1}$ coordinates and $x_{2} \mathrm{O}_{2} z_{2}$ coordinate are established. Among them, $x_{1} \mathrm{O}_{1} z_{1}$ coordinate system is consistent with the directions of $x \mathrm{O} z$ coordinate. However, $x_{2} \mathrm{O}_{2} z_{2}$ corresponds to a transformation that $x \mathrm{o} z$ rotates $\pi$ around $y$-axis. This transformation does not affect the material properties, so the stress parameters do not change.

The radial force $\boldsymbol{F}$ can be regarded as acting on the boundary of semi-infinite plane media when it acts on the thin circular wafer $\mathrm{o}_{1}$ and $\mathrm{o}_{2}$ points. So in $x_{1} \mathrm{o}_{1} \mathrm{z}_{1}, x_{2} \mathrm{o}_{2} z_{2}$ coordinates, the normal stresses $\sigma_{1}$ and $\sigma_{2}$ generated by the force at any point $\mathrm{P}(r, \theta)$ on the circular wafer respectively are:

$$
\left\{\begin{array}{l}
\sigma_{1}=\frac{1}{r_{1}} \cdot \frac{\lambda \cos \theta_{1}+\mu \sin \theta_{1}}{1+\alpha \cos 2 \theta_{1}+\beta \cos 4 \theta_{1}+\gamma \sin 2 \theta_{1}+\delta \sin 4 \theta_{1}} \\
\sigma_{2}=\frac{1}{r_{2}} \cdot \frac{\lambda \cos \theta_{2}-\mu \sin \theta_{2}}{1+\alpha \cos 2 \theta_{2}+\beta \cos 4 \theta_{2}-\gamma \sin 2 \theta_{2}-\delta \sin 4 \theta_{2}}
\end{array}\right.
$$

In the formula, $\lambda, \mu$ are the stress parameters decided by the boundary conditions and force equilibrium conditions; $\alpha, \beta, \gamma, \delta$ are material constants decided by elastic compliance constant tensor matrix elements of the dielectric material (Crystal elastic anisotropy is reflected in the parameters of these materials). For At-cut quartz crystal, the relationship of material constants and $\psi$ is: $\alpha=0.08931 \cos 2 \psi, \beta=0.2062 \cos 4 \psi, \gamma=0.08931 \sin 2 \psi \quad, \delta=0.2062 \sin 4 \psi$; The relationship 
of stress constant $\lambda, \mu, \psi$ and $\mathrm{F}$ is: $\lambda=-4 F(0.4884+0.0181 \cos 2 \psi) / \pi, \quad \mu=-4 F(0.0181 \cos 2 \psi) / \pi$.

When the resonator, that is seen as a force-sensitive element, whose matrix is formed by a thin quartz crystal wafer is fixed in the conversion mechanism, the edge of the resonator is free in addition to the force application point. We image that on the circumference there is an additional stress $f(R, \theta)$ changed with the position exists except that the radial force is applied to the edge of the plane from $\mathrm{o}_{1}, \mathrm{o}_{2}$. That makes the points on the circumference except the points of $\mathrm{o}_{1}$ and $\mathrm{o}_{2}$ meet free boundary condition (i.e., zero stress conditions). Under the action of $\boldsymbol{F}$ and $f(R, \theta)$, circumferential meet free boundary conditions. Thus, the stress at any point within the wafer is the synthetic stress of $\sigma_{1}, \sigma_{2}$ and $f(R, \theta)$.

So in Fig.1, we transform $\sigma_{1}, \sigma_{2}$ of $\mathrm{P}(r, \theta)$ in the polar coordinates, that is the transformation that $\sigma_{1}$ rotates $\psi_{1}=\left(\pi / 2+\theta-\theta_{1}\right)$ around $y$-axis and $\sigma_{2}$ rotates $\psi_{2}=-\left(\pi / 2-\theta-\theta_{2}\right)=\left(\theta+\theta_{2}-\pi / 2\right)$ around $\mathrm{y}$-axis. Then we superpose the components in the same direction and obtain:

$$
\left\{\begin{array}{l}
\sigma_{r}^{\prime}(r, \theta)=\sigma_{1} \sin ^{2}\left(\theta_{1}-\theta\right)+\sigma_{2} \sin ^{2}\left(\theta+\theta_{2}\right) \\
\sigma_{\theta}^{\prime}(r, \theta)=\sigma_{1} \cos ^{2}\left(\theta-\theta_{1}\right)+\sigma_{2} \cos ^{2}\left(\theta+\theta_{2}\right) \\
\tau_{r \theta}^{\prime}(r, \theta)=-\sigma_{1} \sin \left(\theta-\theta_{1}\right) \cos \left(\theta-\theta_{1}\right)-\sigma_{2} \sin \left(\theta+\theta_{2}\right) \cos \left(\theta+\theta_{2}\right)
\end{array}\right.
$$

In order to make the solution of the stress within the wafer satisfy the boundary conditions on the free circumference, the stress at any point $\mathrm{P}(r, \theta)$ on the wafer can be supposed that:

$$
\left\{\begin{array}{l}
\sigma_{r}(r, \theta)=\sigma_{r}^{\prime}(r, \theta)+f_{r}(R, \theta) \\
\sigma_{\theta}(r, \theta)=\sigma_{r}^{\prime}(r, \theta)+f_{\theta}(R, \theta) \\
\tau_{r \theta}(r, \theta)=\tau_{r \theta}^{\prime}(r, \theta)+f_{r \theta}(R, \theta)
\end{array}\right.
$$

Using the free boundary conditions, we can obtain additional compensation function that is irrelevant with r: $f_{r}(R, \theta), f_{\theta}(R, \theta), f_{r \theta}(R, \theta)$.

Thus, according to the formula (1), (2), (3), we can get the points of stress distribution analytical solution $\sigma_{r}(r, \theta), \sigma_{\theta}(r, \theta), \tau_{r \theta}(r, \theta)$ on the AT-cut (including general cut methods) thin circular piece of quartz crystal when it is subjected to a radial concentrated force whose azimuth is $\psi$ and strength is $\boldsymbol{F}$ (along the unit thickness).

\section{The Theoretical Calculations And Test Results}

According to the stress distributions $\sigma_{r}, \sigma_{\theta}$ of the points in the thin round wafer obtained above, the strain $S$ of $\mathrm{P}(0,0)$ which is located in the center of the resonator electrode calculated by Hooke's Law and the relationship between $S$ and the relative change $(\Delta f / f)$ in frequency chip, we can find the relationship between $(\Delta f / f)$ and the adding force azimuth $\psi$ of a thin circular crystal plate 
whose radius is $R$ when it is subjected to a radial force $\boldsymbol{F}$ :

$$
\Delta f / f=\left(\frac{2 F}{\pi R}\right) \bar{S} \times 10^{-12}
$$

In the formula:

$$
\begin{aligned}
\bar{S}= & \left(27.218 \cos ^{2} \psi+0.656 \sin ^{2} \psi\right) \cdot\left[\frac{4(0.4884+0.0181 \cos 2 \psi)}{1+0.0893 \cos 2 \psi+0.2063 \cos 4 \psi}-\frac{(0.4884+0.0181 \cos 2 \psi)(1-0.2063 \cos 4 \psi)-0.00162 \sin ^{2} 2 \psi}{(1-0.2063 \cos 4 \psi)^{2}-0.00797 \sin ^{2} 2 \psi}\right]- \\
& \left(27.218 \sin ^{2} \psi+0.656 \cos ^{2} \psi\right) \cdot \frac{(0.4884+0.0181 \cos 2 \psi)(1-0.2063 \cos 4 \psi)-0.00162 \sin ^{2} 2 \psi}{(1-0.2063 \cos 4 \psi)^{2}-0.00797 \sin ^{2} 2 \psi}- \\
& 26.562 \sin ^{2} 2 \psi \cdot \frac{0.02551+0.00373 \cos 4 \psi+0.00162 \cos 2 \psi}{(1-0.2063 \cos 4 \psi)^{2}-0.00797 \sin ^{2} 2 \psi}
\end{aligned}
$$

For the symmetric single electrode thin circular At-cut quartz crystal resonators, as shown in Fig.2, if the " $(2 F / \pi R) \times 10^{-12 "}$ is a unit, the theoretical curve about the relative change of the resonant frequency ( $\Delta f / f)$ and the adding force azimuth $\psi$ can be calculated by the formula (4), and it is shown in a broken line in Fig.3. In Fig.3, the solid line is the actual test curve of frequency relative change in the At-cut quartz crystal resonator (wafer thickness $d=0.27 \mathrm{~mm}$, radius $R=7 \mathrm{~mm}$, $f=f_{0}=5900 \mathrm{kHz}$, energizing weight $m=72 \times 10^{-3} \mathrm{~kg}, F=m \mathrm{~g} / d=2.613 \times 10^{3} \mathrm{~N} / \mathrm{m}, 2 F / \pi R=0.237 \times 10^{6}$ $\mathrm{N} / \mathrm{m}^{2}$ ). The trend of its theoretical curve and experimental curve is completely similar (The theoretical value is slightly higher about 7\%). So for the single electrode symmetrical quartz crystal resonators, the theoretical calculation obtained by the formula (4) is instructive to the designs, such as the resonator electrode lead and radial force position (i.e., the resonator edge consolidation point) when it uses its power frequency sensitive characteristics.

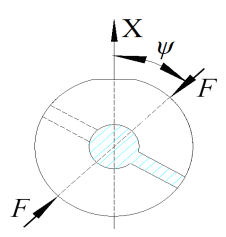

Fig.2 Symmetrical structure quartz crystal resonators



Fig.3 The relationship of the relative change in frequency and the adding force azimuth

\section{Finite Element Analysis And Experimental Comparison}

\section{Finite Element Analysis In The Central Region}

To the thin circular AT-cut quartz crystal in Fig.2, we analyze the stress distribution within the crystal piece by the ANSYS finite element method when it is subject to a radial force $\boldsymbol{F}$. When $\boldsymbol{F}=$ $5 \mathrm{~N}$, and its direction is along the $X$-axis (crystal axis), the stress distribution is shown in Fig.4. The results show that under the same radial force, the change of the stress varies widely in different locations. 


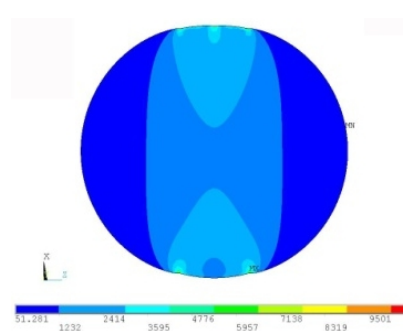

( a )

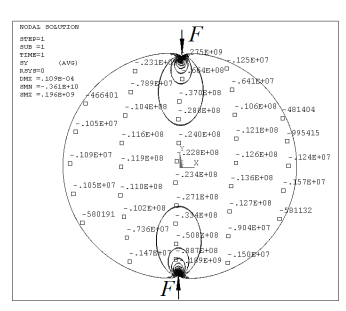

( b )

Fig.4 the stress distribution within the thin circular quartz crystal when the radial force is applied

When the force $\boldsymbol{F}$ is constant and the adding force azimuth $\psi$ changes, the relationship of the stress variation $\Delta \boldsymbol{F}$ and the azimuth $\psi$ in the central area of the wafer (i.e., the electrode central) can be got. It is shown in Fig.5. Within the crystal, the stress change is closely related to the adding force azimuth. And the relationship of their changes is similar to that showed in Fig.3.

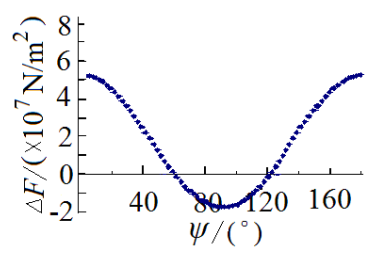

Fig. 5 The relationship between $\Delta F$ and $\psi$ in the central area of a thin wafer

\section{Finite Element Analysis In The Non-central region}

It is difficult to get a clear analytical solution by using the equation (4) to calculate the stress distribution outside the geometric center area of the thin circular wafer. Therefore, we can use ANSYS finite element analysis software to carry out the relevant analysis.

If the resonator electrode (and the analysis points) in asymmetric position of thin circular wafer, as shown as Fig.6 and Fig.8, we can obtain the relationship between the crystal internal stress change $\Delta F$ and the adding force azimuth $\psi$ in the central area of the electrodes under the radial force $\boldsymbol{F}(=5 \mathrm{~N})$. They are respectively shown in Fig.7 and Fig. 9.

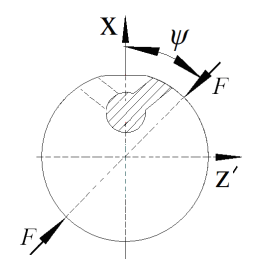

Fig.6 Quartz crystal resonator of asymmetric structure(1)

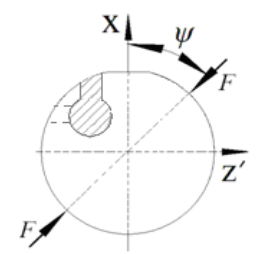

Fig.8 Quartz crystal resonator of asymmetric structure (2)

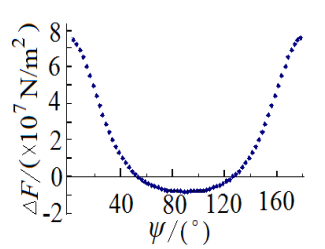

Fig.7 The relationship about $\Delta F-\psi$ in electrode center of asymmetric structure resonator(1)

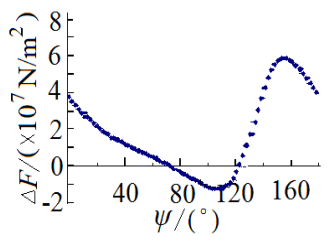

Fig.9 The relationship about $\Delta F-\psi$ in electrode center of asymmetric structure resonator(2) 


\section{The Experimental Comparison}

If the wafer whose diameter is $12.5 \mathrm{~mm}$ and thickness is $0.16 \mathrm{~mm}$ is subjected to radial force, when it is applied to a radial force $\boldsymbol{F}=5 \mathrm{~N}$ along the $X$-axis direction, we can get the stress changes of the central A, B and C whose distance to A is $3 \mathrm{~mm}$ In Fig. 10 by using ANSYS finite element analysis method. The stress changes respectively are: $\Delta \boldsymbol{F}_{\mathrm{A}}=-6.81 \times 10^{7} \mathrm{~N} / \mathrm{m}^{2}, \Delta \boldsymbol{F}_{\mathrm{B}}=-8.99 \times 10^{7}$ $\mathrm{N} / \mathrm{m}^{2} 、 \Delta \boldsymbol{F}_{\mathrm{C}}=-4.07 \times 10^{7} \mathrm{~N} / \mathrm{m}^{2}$

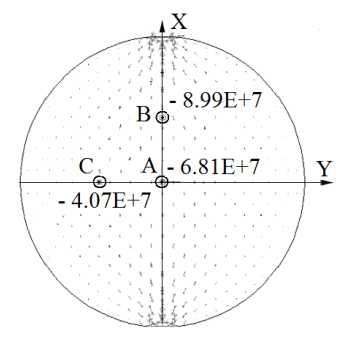

Fig.10 The stress change at different positions within the thin quartz crystal wafer

In Fig.10, the three points $\mathrm{A}, \mathrm{B}$ and $\mathrm{C}$ of the wafer respectively coat electrode to constitute resonators at different positions. And we exert a radial concentrated force along the $X$-axis direction of the crystal and measure resonator frequency variations at the three positions $\mathrm{A}, \mathrm{B}, \mathrm{C}$ by using the frequency meter to obtain corresponding force - frequency conversion coefficient $K$. They respectively are: $K_{\mathrm{A}}=189.9 \mathrm{~Hz} / \mathrm{N} 、 K_{\mathrm{B}}=255.3 \mathrm{~Hz} / \mathrm{N} 、 K_{\mathrm{C}}=115.0 \mathrm{~Hz} / \mathrm{N}$.

The results $\Delta \boldsymbol{F}_{\mathrm{A}}, \Delta \boldsymbol{F}_{\mathrm{B}}, \Delta \boldsymbol{F}_{\mathrm{C}}$ obtained by ANSYS analysis and the experimental results $K_{\mathrm{A}}, K_{\mathrm{B}}$ 、 $K_{\mathrm{C}}$ will be processed numerically as follows:

$$
\begin{aligned}
& \eta_{\mathrm{A}}=\left|\Delta F_{\mathrm{A}} / K_{\mathrm{A}}\right|=3.58 \times 10^{5} \\
& \eta_{\mathrm{B}}=\left|\Delta F_{\mathrm{B}} / K_{\mathrm{B}}\right|=3.52 \times 10^{5} \\
& \eta_{\mathrm{C}}=\left|\Delta F_{\mathrm{C}} / K_{\mathrm{C}}\right|=3.54 \times 10^{5}
\end{aligned}
$$

We compare $\eta_{\mathrm{A}}, \eta_{\mathrm{B}}, \eta_{C}$ obtained above and find that their corresponding values are basically consistent. The maximum relative error is about $1.7 \%$.

The results indicated that the crystal stress distribution obtained by ANSYS analysis corresponds to the force-sensitive character in the correspond positions and the resonator's force-sensitive character is related to the location of the electrode with centralized force to the crystal. Therefore, if a lot of resonators are designed on the same crystal substrate, it can use the method of finite element analysis in the limited space scales to seek the position of relatively large changes in stress difference and to set the resonator in the correspond location. They output in the form of the difference frequency between the resonators. It can get integrated quartz crystal resonators whose force-sensitive character is obvious.

\section{Conclusion}

Extending the stress in semi-infinite anisotropic media slice with centralized force to thin circular 
quartz crystal flake, the relationship of frequency relative variation and adding force azimuth and adding force strength of the crystal with radial centralized force was obtained by the method of additional stress, i.e., force-sensitive character. And It has been verified experimentally; In the position deviated from geometric center, the stress variation obtained by the finite element method is consistent with the resonator's force-sensitive character after adding electrode. The theoretical calculations and ANSYS analysis laid the foundation for the design of multi-electrode resonator based on the same crystal wafer.

\section{Acknowledgements}

This work was supported by National Natural Science Foundation of China (60968001), Beijing Natural Science Foundation (4122030), Beijing natural science foundation of key projects (B) (KZ201511232037), Key Laboratory of Modern Measurement \& Control Technology (Beijing Information Science \& Technology University), Ministry of Education.

\section{Biography:}

Tian Wenjie (1962-), male, Zhaotong of Yunnan province, Professor, Ph.D., the major is quartz inertial device research and teaching work.

\section{References}

[1] RATAJSKIM J M. Force-frequency coefficient of single rotated vibrating quartz crystals [J].J.Res. Dev. BM.1968,12(1):92-99

[2] Eernisse E P, Lukaszek T, Ballat A. Variational calculation of force-frequency constants of doubly rotated quartz resonators[J]. IEEE Trans Sonics Ultrason, 1978, SU-25:132-138.

[3] Wang Z Y, Zhu H Z, Dong Y G. Force-frequency coefficient of symmetrical incomplete circular quartz crystal resonator [J] . IEEE Tran Ultrason, ferroelectrics, and frequency control, 2001, 48: 1471-1479.

[4] Malov. B.B. piezoelectric resonant sensor [M]. translated by Weng Shanchen. Beijing: National Defense Industry Press, 1-237 1984

[5]M. Ferrari, V. Ferrari, K.K. Kanazawa . Dual-harmonic oscillator for quartz crystal resonator sensors [J].Sensors and Actuators A: Physical, Vol. 145-146(2008)131-138

[6]Ji Wang, Wenhua Zhao, Jianke Du, Yuantai $\mathrm{Hu}$.The calculation of electrical parameters of AT-cut quartz crystal resonators with the consideration of material viscosity[J]. Ultrasonics, Vol.51( 1)( 2011) 65-70

[7]Ma Jing, Zhibin Wang. Analysis on Temperature- frequency Characteristic of Quartz Resonator[J] .Silicon Valley. 2012,1:54.

[8] LEE P C Y,WANG Y S,MARKENSCOFF X. High -frequency vibrations of crystal plates under initial stresses [J]. J. Acoust. Soc. Am. , 1975.57(1):96-105

[9] JANIAUD D,NISSIM L,GAGGNEPAIN J J. analytic calculation of initial stress effects on anisotropic crystals: Application to quartz resonators [J], Proc.32,AFCS.1978: 169-179. 